TRANSACTIONS OF THE

AMERICAN MATHEMATICAL SOCIETY

Volume 365, Number 11, November 2013, Pages 5655-5680

S 0002-9947(2013)05649-8

Article electronically published on June 26, 2013

\title{
TRANSLATING SOLUTIONS TO LAGRANGIAN MEAN CURVATURE FLOW
}

\author{
ANDRÉ NEVES AND GANG TIAN
}

\begin{abstract}
We prove some non-existence theorems for translating solutions to Lagrangian mean curvature flow. More precisely, we show that translating solutions with an $L^{2}$ bound on the mean curvature are planes and that almostcalibrated translating solutions which are static are also planes. Recent work of D. Joyce, Y.-I. Lee, and M.-P. Tsui shows that these conditions are optimal.
\end{abstract}

\section{INTRODUCTION}

It was shown in $[5$ that finite-time singularities are, in some sense, unavoidable. More precisely, the first author gave examples of Lagrangians in $\mathbb{C}^{2}$ having the Lagrangian angle as small as we want and for which the Lagrangian mean curvature flow develops a finite-time singularity. Thus, if one aims to use Lagrangian mean curvature flow in order to understand the existence problem for Special Lagrangians (i.e. Lagrangians which are minimal surfaces), it is crucial to understand how finitetime singularities form. The next example shows that this is a rather non-trivial problem.

Example 1.1. Let $\gamma_{0}$ be the curve in $\mathbb{C}$ given in Figure 1

The curve can be made so that, under curve-shortening flow $\left(\gamma_{t}\right)_{t>0}$, the small loop collapses at time $T$ and $\gamma_{T}$ becomes a curve with a cusp point. Moreover, $\gamma_{0}$ can be chosen so that the angle the tangent vector makes with the $x$-axis has an oscillation not much larger than $\pi$. Let $L_{t}$ be the Lagrangian surface in $\mathbb{C}^{2}$ given by

$$
L_{t}=\gamma_{t} \times \mathbb{R} \subset \mathbb{C} \times \mathbb{C} .
$$

Then, $L_{0}$ is a zero-Maslov class Lagrangian with oscillation of the Lagrangian angle as close to $\pi$ as we want, which develops a singularity at time $T$. The singular set is a line of cusp-points and hence has Hausdorff dimension one.

This example shows that in order to develop a regularity theory for the flow, i.e., show that singularities for Lagrangian mean curvature flow are isolated, we need to require the oscillation of the Lagrangian angle to be strictly smaller than $\pi$ (almost-calibrated).

So far, the only known evidence that such regularity theory is possible was given in [5]. There, assuming the initial condition is a rational and almost-calibrated Lagrangian, the first author showed that if one rescales the flow around a fixed point in space-time, connected components of this rescaled flow converge to an area-minimizing union of planes. The fact that the rescaled flow converges to a

Received by the editors June 9, 2011.

2010 Mathematics Subject Classification. Primary 53C44, 53D12.

The author was partially supported by NSF grant DMS-06-04164.

(C)2013 American Mathematical Society 


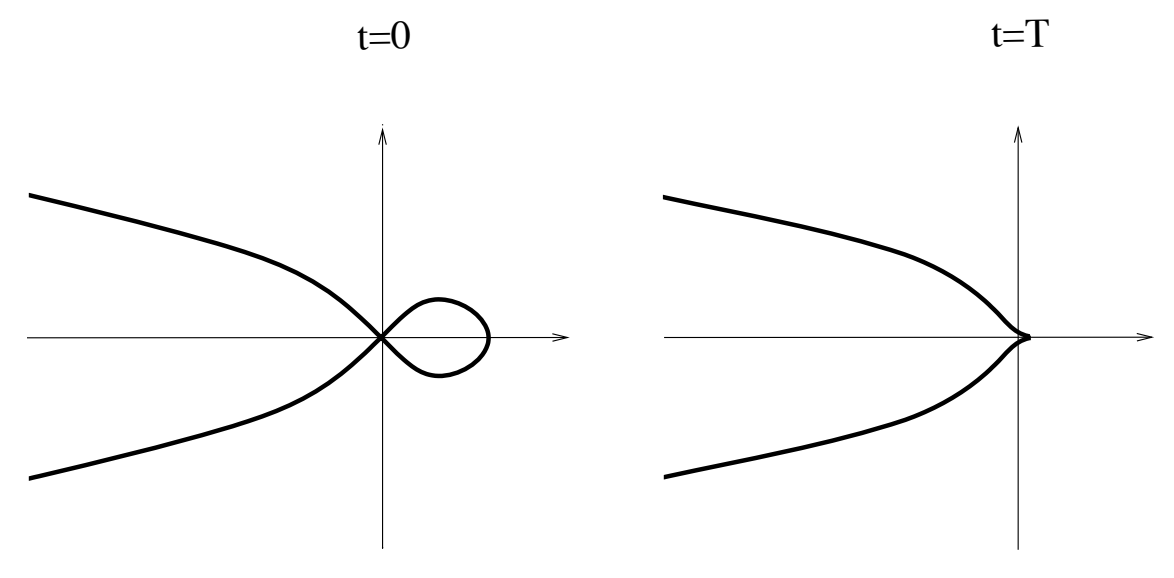

Figure 1. Curves $\gamma_{0}$ and $\gamma_{T}$.

union of planes is an almost trivial consequence of Huisken's monotonicity formula, and so the interesting part is that the configuration of planes needs to be areaminimizing. Without this property it would be hopeless to expect any regularity theory.

Nonetheless, we should point out that the property mentioned above is not sufficient to develop a regularity theory. One needs to understand dilations of the flow where the point at which we center the dilation changes with the scale (called Type II dilations). From general theory, it follows that Type II dilations converge to an eternal solution with second fundamental form uniformly bounded. If singularities are indeed isolated, the expectation is that this eternal solution has vanishing mean curvature. We now describe heuristically what could happen regarding Example 1.1. If we rescale around the fixed point in space-time at which the singularity is developing, the rescaled flow converges to a plane with multiplicity two. On the other hand, if we rescale the flow around the point of highest second fundamental form at some time $t_{1}$ close to $T$ and choose the scale so that the second fundamental form for the new solution becomes bounded by one, the rescaled flow converges to the eternal solution given by

$$
L_{t}=\left\{\left(-\log \cos y_{1}+t, y_{1}, x_{2}, 0\right) \mid-\pi / 2<y_{1}<\pi / 2, x_{2} \in \mathbb{R}\right\} .
$$

This solution is called the grim-reaper and is an example of translating solutions to mean curvature flow.

Definition 1.2. A Lagrangian $L$ is a translating solution to Lagrangian mean curvature flow if we can find an ambient vector $e_{1}$ so that

$$
L_{t}=L+t e_{1}
$$

is a solution to mean curvature flow.

Remark 1.3. Without loss of generality, we assume that $e_{1}=(1,0,0,0)$. We can always achieve this by scaling $L$ and then choosing a suitable coordinate system.

In a surprising new result, Dominic Joyce, Yng-Ing Lee, and Mao-Pei Tsui [4] found translating solutions to Lagrangian mean curvature flow with oscillation of 
the Lagrangian angle arbitrarily small. They are described as follows. Let $w$ be a curve in $\mathbb{C}$ such that

$$
w_{t}=\sqrt{2 t} w \text { for } t>0
$$

is a solution to curve shortening flow in $\mathbb{C}$. This curve can be chosen so that the angle $\theta$ that the tangent vector makes with the $x$-axis has arbitrarily small oscillation. Set

$$
L=\left\{\left(\frac{|w|^{2}(y)-x^{2}}{2}-i \theta(y), x w(y)\right) \mid x, y \in \mathbb{R}\right\} \subset \mathbb{C} \times \mathbb{C} .
$$

Using the fact that the curvature of $w$ satisfies

$$
\vec{k}=w^{\perp},
$$

it is a straightforward computation to check that $L$ is Lagrangian and that

$$
L_{t}=L+t(1,0,0,0)
$$

is a solution to Lagrangian mean curvature flow. Moreover, the Lagrangian angle of $L$ coincides with $\theta$, and hence its oscillation can be made arbitrarily small.

The main purpose of this paper is to give conditions that exclude the existence of non-trivial translating solutions to Lagrangian mean curvature flow. In order to do so, we need one more definition.

Let $\left(L_{t}\right)_{-\infty<t<\infty}$ be an eternal solution to Lagrangian mean curvature flow in $\mathbb{C}^{2}$ which is almost-calibrated. Given a sequence $\left(\lambda_{i}\right)_{i \in \mathbb{N}}$ converging to zero, we can consider the sequence of blow-downs

$$
L_{s}^{i}=\lambda_{i} L_{s / \lambda_{i}^{2}}, \quad \text { where }-\infty<s<\infty .
$$

It follows from Theorem 3.1 that we can extract a subsequence $L_{s}^{i}$ converging weakly to the same union of planes for every $s \leq 0$, which we denote by $L^{\infty}$ (see Theorem 3.1 for the notion of weak convergence we are considering).

Definition 1.4. An eternal solution to Lagrangian mean curvature flow is called static if we can find a convergent sequence of blow-downs $L_{s}^{i}$ that converges in the Radon measure sense to $L^{\infty}$ for every $s>0$.

In Theorem 3.1 we characterize the blow-downs of eternal solutions. In particular, it will be seen in Theorem 3.1 that assuming $L$ is exact (see the next section for a definition) and $\inf _{L} \cos \theta>0$, where $\theta$ denotes the Lagrangian angle, then if $L_{s_{0}}^{i}$ converges to a union of planes for some $s_{0}>0, L_{s}^{i}$ must converge to $L^{\infty}$ for every $s>0$. The reason is that in that case the limit of $L_{s_{0}}^{i}$ will be stationary, and thus the last statement of Theorem 3.1 applies.

Theorem A. Let $L$ be a translating solution for Lagrangian mean curvature flow in $\mathbb{C}^{2}$ for which we can find a constant $C_{1}$ such that

$$
\left\{\begin{array}{l}
\text { The first Betti number of } L \text { is finite, } \\
\sup _{L}|\theta| \leq C_{1}, \\
\mathcal{H}^{2}\left(L \cap B_{R}(0)\right) \leq C_{1} R^{2} \quad \text { for all } R>0 \\
\sup _{L}|A|^{2} \leq C_{1} .
\end{array}\right.
$$

If

$$
\int_{L}|H|^{2} d \mu \quad \text { is finite }
$$


or if

$$
L \text { is static and } \inf _{L} \cos \theta \geq \varepsilon_{1}
$$

for some $\varepsilon_{1}>0$, then $L$ is a plane.

Remark 1.5. (1) $n=2$ is necessary for the following reason. Special Lagrangians which are invariant under translation by $e_{1}$ are translating solutions. For instance, if $L$ is any Special Lagrangian in $\mathbb{C}^{2}$, then $\tilde{L}=\mathbb{R} \times L \subset$ $\mathbb{C}^{3}$ is also a translating solution, and so the theorem is false when $n=3$. It is a simple fact that smooth Special Lagrangians in $\mathbb{C}^{2}$ which are invariant under $e_{1}$ must be a plane, and this fact will be used in the proof of Theorem 4.3

(2) The static condition is necessary for the following reason. Consider the translating solution $\left(L_{t}\right)_{-\infty<t<\infty}$ described in (2) and denote by $\tilde{w}$ the curve in $\mathbb{C}$ given by the union of $w$ with $-w$. If $\left(\lambda_{i}\right)_{i \in \mathbb{N}}$ is a sequence converging to zero, the curves $\sqrt{\lambda_{i}} \tilde{w}$ converge to a union of two lines crossing at the origin which we denote by $\tilde{w}_{0}$. A simple computation shows that the sequence of blow-downs $L_{s}^{i}$ converges to the union of two planes given by

$$
\mathbb{R} \times \tilde{w}_{0} \subseteq \mathbb{C} \times \mathbb{C}
$$

for every $s \leq 0$ and to a self-expander given by

$$
\sqrt{2 s}(\mathbb{R} \times \tilde{w})=\mathbb{R} \times \sqrt{2 s} \tilde{w} \subseteq \mathbb{C} \times \mathbb{C}
$$

for every $s>0$.

(3) The condition

$$
\inf _{L} \cos \theta \geq \varepsilon_{1}
$$

is necessary because otherwise the grim-reaper described in (11) would be a counterexample.

The paper is organized as follows. In Section 2 we introduce some basic notation and derive some simple identities for translating solutions, and in Section 3 we prove a compactness theorem for the blow-down sequence of an eternal solution.

In Section 4 we assume that, outside a compact set, $L$ can be decomposed into $N$ components $L_{1}, \ldots, L_{N}$, where each $L_{j}$ is the graph of a multi-valued function defined over a plane $P_{j}$ minus a disc, and show that in this case $L$ needs to be a plane (Theorem 4.3). The argument consists of using barriers to show that on each component $L_{j}$ the Lagrangian angle converges to a constant sufficiently fast, and this will imply that, by choosing $R$ sufficiently large, $L_{j} \backslash B_{R}(0)$ can be made as close to a plane as we want. In particular,

$$
\lim _{R \rightarrow \infty} \oint_{\partial\left(L \cap B_{R}(0)\right)}\left\langle\nu, e_{1}\right\rangle d \sigma=0
$$

where $\nu$ denotes the exterior unit normal to $\partial\left(L \cap B_{R}(0)\right)$ in $L$. The result will follow because Proposition 2.2 (ii) implies that

$$
\oint_{\partial\left(L \cap B_{R}(0)\right)}\left\langle\nu, e_{1}\right\rangle d \sigma=\int_{L \cap B_{R}(0)} \Delta x_{1} d \mu=\int_{L \cap B_{R}(0)}|H|^{2} d \mu,
$$

and so $L$ will have zero mean curvature. In that section we also prove a lemma (Lemma 4.6) that gives us conditions under which a translating solution to Lagrangian mean curvature flow admits a graphical decomposition. 
In Section 5 we show that if $L$ has the $L^{2}$-norm of $|H|$ bounded or if $L$ is static and almost-calibrated, then $L$ satisfies the conditions specified in Lemma 4.6 and hence admits a graphical decomposition.

1.1. Open questions. We propose two questions whose answers could provide some valuable insight as to whether it is reasonable to expect any good behavior for singularities of Lagrangian mean curvature flow.

The first question is whether the translating solutions found by D. Joyce, Y.-I. Lee, and M.-P. Tsui can arise as a blow-up of a finite time singularity for Lagrangian mean curvature flow.

If one aims to develop a regularity theory for the flow, it is absolutely necessary to answer this question. This relation has been observed before in codimension one mean curvature flow and Ricci flow. In the first case, Brian White used his work on mean convex solutions to mean curvature flow 9 to show that no grim-reaper appears as the limit of a sequence of rescaled flows [10, Corollary 4]. In the second case, one of the first breakthroughs of Perelman [ $[$, Section 4] was to show that the cigar soliton does not arise as a finite-time singularity model.

If one could show that, assuming the initial condition for the flow is an exact and almost-calibrated Lagrangian, no blow-down of a Type II rescale can give rise to a non-stationary self-expander, then, in view of Theorem 3.1, the question above would be solved for a large class of initial conditions.

The second question addresses the issue of uniqueness of translating solutions. Suppose that $\left(L_{t}\right)_{t \in \mathbb{R}}$ and $\left(L_{t}^{\prime}\right)_{t \in \mathbb{R}}$ are two translating solutions which are almost calibrated and exact. If after blow-down they produce the same self-expander, do they have to differ only by a rigid motion?

\section{Definitions And BASiC identities}

Let $J$ and $\omega$ denote, respectively, the standard complex structure on $\mathbb{C}^{2}$ and the standard symplectic form on $\mathbb{C}^{2}$. We also consider the closed complex-valued 2 -form given by

$$
\Omega \equiv d z_{1} \wedge d z_{2},
$$

where $z_{j}=x_{j}+i y_{j}$ are complex coordinates of $\mathbb{C}^{2}$, and the Liouville form

$$
\lambda=\sum_{j=1}^{2} x_{j} d y_{j}-y_{j} d x_{j} .
$$

We denote by $\mathcal{H}^{1}$ and $\mathcal{H}^{2}$ the one dimensional and two dimensional Hausdorff measures in $\mathbb{C}^{2}$, respectively.

A smooth two-dimensional submanifold $L$ in $\mathbb{C}^{2}$ is said to be Lagrangian if $\omega_{L}=$ 0 , and this implies that

$$
\Omega_{L}=e^{i \theta} \operatorname{vol}_{L},
$$

where $\operatorname{vol}_{L}$ denotes the volume form of $L$ and $\theta$ is a multi-valued function called the Lagrangian angle. When the Lagrangian angle is a single-valued function the Lagrangian is called zero-Maslov class, and if

$$
\cos \theta \geq \varepsilon_{0}
$$


for some positive $\varepsilon_{0}$, then $L$ is said to be almost-calibrated. Furthermore, if $\theta \equiv \theta_{0}$, then $L$ is calibrated by

$$
\operatorname{Re}\left(e^{-i \theta_{0}} \Omega\right)
$$

and hence area-minimizing. In this case, $L$ is referred to as being Special Lagrangian. The Lagrangian $L$ is said to be exact if the Liouville form is an exact form on $L$.

Finally, the relation between the Lagrangian angle and the mean curvature is given by

$$
H=J \nabla \theta
$$

Given a point $x_{0}$ in $\mathbb{C}^{2}$ and a time $T$, the backwards heat kernel is defined as

$$
\Phi_{x_{0}, T}(x, t)=\frac{\exp \left(-\frac{\left|x-x_{0}\right|^{2}}{4(T-t)}\right)}{4 \pi(T-t)} .
$$

When $x_{0}$ is the origin and $T=0$, we denote it by $\Phi$. When it is clear from the context at which instant $t$ we are evaluating $\Phi_{x_{0}, T}(x, t)$, we denote it simply by $\Phi_{x_{0}, T}$. Moreover, $\mathbf{x}$ and $\mathbf{x}^{\perp}$ stand for the position vector associated with the point $x$ in $\mathbb{C}^{2}$ and its projection on the normal space of $T_{x} L$, respectively.

Lemma 2.1 (Huisken's monotonicity formula). Let $f_{t}$ be a smooth family of functions in $L_{t}$. Then

$$
\frac{d}{d t} \int_{L_{t}} f_{t} \Phi_{x_{0}, T} d \mu=\int_{L_{t}}\left(\partial_{t} f_{t}-\Delta f_{t}\right) \Phi_{x_{0}, T} d u-\int_{L_{t}}\left|H+\frac{\left(\mathbf{x}-x_{0}\right)^{\perp}}{2\left(T-t_{0}\right)}\right|^{2} \Phi_{x_{0}, T} d \mu .
$$

Throughout this paper, $\left(L_{t}\right)_{-\infty<t<\infty}$ will be a translating solution to Lagrangian mean curvature flow in $\mathbb{C}^{2}$, where $L$ satisfies hypothesis $(H)$. We denote by $\left|e_{1}^{\perp}\right|$ the projection of $e_{1}=(1,0,0,0)$ on the normal space of $L$. The intrinsic ball of radius $r$ around a point $x$ in $L$ is defined by $\widehat{B}_{r}(x)$, and we fix $r_{0}<1$ to be such that $\widehat{B}_{r_{0}}(x)$ is simply connected for every $x$ in $L$.

Proposition 2.2. The following equations hold on $L$.

(i) There is a constant $\theta_{0}$ such that

$$
\theta(x)=-\left\langle J e_{1}, \mathbf{x}\right\rangle+\theta_{0} \quad \text { and } \quad|H|=\left|e_{1}^{\perp}\right| ;
$$

$$
\Delta x_{1}=|H|^{2}
$$

$$
\Delta \theta+\left\langle\nabla \theta, e_{1}\right\rangle=0
$$

Proof. Because $\left(L_{t}\right)_{-\infty<t<\infty}$ is a translating solution to mean curvature flow we have that

and thus

$$
H=e_{1}^{\perp},
$$

$$
\nabla \theta=-J e_{1}^{\perp}=-\left(J e_{1}\right)^{\top} .
$$

This implies the first property. The second one follows from

$$
\Delta x_{1}=\left\langle H, e_{1}\right\rangle=|H|^{2},
$$

and the third property is a consequence of

$$
\Delta \theta=-\left(J e_{1}\right)^{\top}=\left(J e_{1}\right)^{\perp}=-\left\langle H, J e_{1}\right\rangle=-\left\langle\nabla \theta, e_{1}\right\rangle .
$$


Given a sequence $\left(\lambda_{i}\right)_{i \in \mathbb{N}}$ converging to zero, we define the sequence of blowdowns

$$
L_{s}^{i}=\lambda_{i} L_{s / \lambda_{i}^{2}}, \quad \text { where } \quad-\infty<s<\infty .
$$

Finally, we use the notation

$$
\{\theta=\alpha\}=\{x \in L \mid \theta(x)=\alpha\}
$$

and

$$
\{|\theta-\alpha| \leq \delta\}=\{x \in L|| \theta(x)-\alpha \mid \leq \delta\}
$$

\section{BLOW-DOWN THEOREM}

Let $\left(\Sigma_{t}\right)_{-\infty<t<\infty}$ be an eternal solution to Lagrangian mean curvature flow for which we can find a constant $D$ such that, for every $t$,

$$
\left\{\begin{array}{l}
\mathcal{H}^{2}\left(\Sigma_{t} \cap B_{R}(0)\right) \leq D R^{2} \text { for all } R>0, \\
\cos \left(\theta_{t}\right) \geq D^{-1} .
\end{array}\right.
$$

Given a sequence $\left(\lambda_{i}\right)_{i \in \mathbb{N}}$ converging to zero, consider the blow-downs

$$
\Sigma_{s}^{i}=\lambda_{i} \Sigma_{s / \lambda_{i}^{2}} \text { for }-\infty<s<\infty
$$

Theorem 3.1. There exist a finite set

$$
\left\{\bar{\theta}_{1}, \ldots, \bar{\theta}_{N}\right\}
$$

and Lagrangian planes

$$
P_{1}, \ldots, P_{N}
$$

such that, after passing to a subsequence, we have for every smooth function $\phi$ compactly supported, every $f$ in $C^{2}(\mathbb{R})$, and every $s \leq 0$

$$
\lim _{i \rightarrow \infty} \int_{\Sigma_{s}^{i}} f\left(\theta_{i, s}\right) \phi d \mu=\sum_{j=1}^{N} m_{j} f\left(\bar{\theta}_{j}\right) \int_{P_{j}} \phi d \mu,
$$

where $m_{j}$ denotes the multiplicity of $P_{j}$. The set

$$
\left\{\bar{\theta}_{1}, \ldots, \bar{\theta}_{N}\right\}
$$

does not depend on the sequence of rescales chosen.

If $\Sigma_{0}$ is exact, there exists an integer rectifiable 2 -varifold $\Sigma_{1}^{\infty}$ satisfying

$$
H=\frac{\mathbf{x}^{\perp}}{2}
$$

such that, after passing to a subsequence, $\Sigma_{s}^{i}$ converges as Radon measures to $\sqrt{s} \Sigma_{1}^{\infty}$ for every $s>0$.

If $\Sigma_{1}^{\infty}$ is stationary, then identity (3) holds for every $s$, and hence $\left(\Sigma_{t}\right)_{-\infty<t<\infty}$ is static.

Remark 3.2. (i) Whenever identity (3) holds we say that $\Sigma_{s}^{i}$ converges weakly to $\Sigma^{\infty}=m_{1} P_{1}+\cdots+m_{N} P_{N}$.

(ii) From (3) it follows that the integers $m_{j}$ do not depend on the subsequence chosen. The planes $P_{j}$ could, in principle, depend on the sequence chosen. 
(iii) If one blows-down the grim-reaper defined in Section 1, we see that the limit flow $\left(L_{s}^{\infty}\right)_{s \in \mathbb{R}}$ is a line with multiplicity two for $s<0$, half-line with multiplicity two if $s=0$, and the empty set if $s>0$. Hence, equation (3) holds only for $s<0$ and does not extend to $s=0$. This implies that being almost-calibrated is essential to showing that (3) holds when $s=0$.

Proof. From the compactness for integral Brakke motions [2, Section 7.1] we know that, after passing to a subsequence, $\left(\sum_{s}^{i}\right)_{-\infty<s<\infty}$ converges to an integral Brakke motion $\left(\Sigma_{s}^{\infty}\right)_{-\infty<s<\infty}$. Arguing as in the proof of [5, Theorem A], it is straightforward to see the existence of Lagrangian planes

$$
P_{1}, \ldots, P_{N}
$$

each having multiplicity $m_{i}$ and Lagrangian angle $\bar{\theta}_{i}$, such that (3) holds for every $s<0$. In particular, $\Sigma_{-1}^{\infty}=m_{1} P_{1}+\cdots+m_{N} P_{N}$.

We now argue that (3) also holds when $s=0$. The main technical issue is trying to overcome the fact that $\Sigma_{0}^{i}$ could converge to $\Sigma_{0}^{\infty}$ only in the Radon measure sense and not in the varifold sense.

Federer and Fleming compactness for integral currents implies that, after passing to a subsequence, $\Sigma_{0}^{i}$ converges to an integral current $\Sigma$. We claim that, after rearranging the labeling of the Lagrangian planes $\left\{P_{1}, \ldots, P_{N}\right\}$, there is $Q \leq N$ and $n_{i} \leq m_{i}, i=1, \ldots, Q$, such that the varifold associated to the integral current $\Sigma$ (which we still denote by $\Sigma$ ) coincides with $n_{1} P_{1}+\cdots+n_{Q} P_{Q}$.

We start by remarking that for every $\phi \geq 0$,

$$
\int_{\Sigma_{0}^{\infty}} \phi d \mu \leq D \lim _{i \rightarrow \infty} \int_{\Sigma_{0}^{i}} \phi \cos \theta d \mu=D \lim _{i \rightarrow \infty} \int_{\Sigma_{0}^{i}} \phi \operatorname{Re} \Omega=D \int_{\Sigma} \phi \operatorname{Re} \Omega
$$

and

$$
D^{-1} \int_{\Sigma} \phi d \mu \leq \int_{\Sigma} \phi \operatorname{Re} \Omega=\lim _{i \rightarrow \infty} \int_{\Sigma_{0}^{i}} \phi \operatorname{Re} \Omega \leq \lim _{i \rightarrow \infty} \int_{\Sigma_{0}^{i}} \phi d \mu=\int_{\Sigma_{0}^{\infty}} \phi d \mu,
$$

and so the support of $\Sigma$ coincides with the support of $\Sigma_{0}^{\infty}$. From Huisken's monotonicity formula, Lemma 2.1, we have that, for every $x_{0}$ in $\mathbb{C}^{2}$, every positive $T$, and every $s<0$,

$$
\int_{\Sigma_{0}^{i}} \Phi_{x_{0}, T}(\cdot, 0) d \mu \leq \int_{\Sigma_{s}^{i}} \Phi_{x_{0}, T}(\cdot, s) d \mu
$$

and thus

$$
\int_{\Sigma_{0}^{\infty}} \Phi_{x_{0}, T}(\cdot, 0) d \mu \leq \sum_{j=1}^{N} m_{j} \int_{P_{j}} \Phi_{x_{0}, T}(\cdot, s) d \mu .
$$

Denote the density function of $\Sigma_{0}^{\infty}$ and $\Sigma_{-1}^{\infty}$ by $\Theta_{0}(x)$ and $\Theta(x)$, respectively. Make $T$ go to zero so that the left-hand side converges to $\Theta_{0}\left(x_{0}\right)$ for almost all $x_{0}$. After that, make $s$ converge to zero to obtain $\Theta\left(x_{0}\right) \geq \Theta_{0}\left(x_{0}\right)$, and so the support of $\Sigma$ is contained in the support of $\Sigma_{-1}^{\infty}$. Because $\partial \Sigma=0$, the Constancy Theorem [7, Theorem 26.27] implies, after rearranging the labeling of the Lagrangian planes, the existence of $Q \leq N$ such that the support of $\Sigma$ consists of a union of planes $P_{1}$ through $P_{Q}$. Thus, one can find integers $r_{i} \leq m_{i}$ and $n_{i}, i=1, \ldots, Q$, such that

$$
\Sigma_{0}^{\infty}=r_{1} P_{1}+\cdots+r_{Q} P_{Q} \quad \text { and } \quad \Sigma=n_{1} P_{1}+\cdots+n_{Q} P_{Q} .
$$


If we show that $n_{i} \leq r_{i}$, the claim follows. It suffices to show that $n_{1} \leq r_{1}$. Without loss of generality we assume that the Lagrangian angle of $P_{1}$ equals zero. We have

$$
\begin{aligned}
& n_{1} \int_{P_{1}} \Phi_{x_{0}, T}(\cdot, 0) d \mu \leq \int_{\Sigma} \Phi_{x_{0}, T}(\cdot, 0) \operatorname{Re} \Omega \\
&=\lim _{i \rightarrow \infty} \int_{\Sigma_{0}^{i}} \Phi_{x_{0}, T}(\cdot, 0) \operatorname{Re} \Omega=\lim _{i \rightarrow \infty} \int_{\Sigma_{0}^{i}} \Phi_{x_{0}, T}(\cdot, 0) \cos \theta d \mu \\
& \quad \leq \lim _{i \rightarrow \infty} \int_{\Sigma_{0}^{i}} \Phi_{x_{0}, T}(\cdot, 0) d \mu=\int_{\Sigma_{0}^{\infty}} \Phi_{x_{0}, T}(\cdot, 0) d \mu .
\end{aligned}
$$

Choosing $x_{0}$ in $P_{1}$ but not in any other $P_{j}$ and making $T$ tend to zero, we conclude that $n_{1} \leq \Theta_{0}\left(x_{0}\right)=r_{1}$.

Next, we show that $\Sigma_{0}^{\infty}$ coincides with $\Sigma_{-1}^{\infty}$. We know that

$$
\frac{d \cos \theta_{t}}{d t}=\Delta \cos \theta_{t}+\cos \theta_{t}|H|^{2},
$$

and thus, for every $T>0$ and $s<0$, we have from the monotonicity formula of Lemma 2.1 that

$$
\begin{aligned}
\left|\frac{d}{d s} \int_{\Sigma_{s}^{i}} \cos \theta_{i, s} \Phi_{0, T} d \mu\right| & \\
=\mid \int_{\Sigma_{s}^{i}} \cos \theta_{i, s} & \left(|H|^{2}-\left|H+\frac{\mathbf{x}^{\perp}}{2(T-s)}\right|^{2}\right) \Phi_{0, T} d \mu \mid \\
& \leq \int_{\Sigma_{s}^{i}} \cos \theta_{i, s}\left(\delta|H|^{2}+C\left|\frac{\mathbf{x}^{\perp}}{2(T-s)}\right|^{2}\right) \Phi_{0, T} d \mu,
\end{aligned}
$$

where $C=C(\delta)$. Combining the evolution equation

$$
\frac{d \theta_{t}^{2}}{d t}=\Delta \theta_{t}^{2}-2|H|^{2}
$$

with Huisken's monotonicity formula, Lemma 2.1, we have that for all $s<0$,

$$
\begin{aligned}
\lim _{i \rightarrow \infty} \int_{s}^{0} \int_{\Sigma_{t}^{i}} 2|H|^{2} \Phi_{0, T} d \mu d t \leq & \lim _{i \rightarrow \infty} \int_{\Sigma_{s}^{i}} \theta_{i, s}^{2} \Phi_{0, T} d \mu \\
& =\sum_{j=1}^{N} m_{j} \bar{\theta}_{j}^{2} \int_{P_{j}} \Phi_{0, T}(\cdot, s) d \mu=\sum_{j=1}^{N} m_{j} \bar{\theta}_{j}^{2}=B .
\end{aligned}
$$

Moreover,

$$
\lim _{i \rightarrow \infty} \int_{s}^{0} \int_{\Sigma_{t}^{i}}\left|\frac{\mathbf{x}^{\perp}}{2(T-t)}\right|^{2} \Phi_{0, T} d \mu d t=0
$$

and so

$$
\lim _{i \rightarrow \infty}\left|\int_{s}^{0} \frac{d}{d t} \int_{\Sigma_{t}^{i}} \cos \theta_{i, t} \Phi_{0, T} d \mu d t\right| \leq \delta \frac{B}{2}
$$


for every $\delta>0$. Thus, making $\delta$ tend to zero, we have

$$
\begin{aligned}
\sum_{j=1}^{Q} n_{j} \cos \bar{\theta}_{j} & =\int_{\Sigma} \Phi_{0, T}(\cdot, 0) \operatorname{Re} \Omega \\
& =\lim _{i \rightarrow \infty} \int_{\Sigma_{0}^{i}} \cos \theta_{i, 0} \Phi_{0, T} d \mu=\lim _{i \rightarrow \infty} \int_{\Sigma_{s}^{i}} \cos \theta_{i, s} \Phi_{0, T} d \mu \\
& =\sum_{j=1}^{N} m_{j} \cos \bar{\theta}_{j} \int_{P_{j}} \Phi_{0, T}(\cdot, s) d \mu=\sum_{j=1}^{N} m_{j} \cos \bar{\theta}_{j} .
\end{aligned}
$$

The fact that $n_{j} \leq m_{j}$ implies that $n_{j}=m_{j}$ and $Q=N$. Because $n_{j} \leq r_{j} \leq m_{j}$, the claim follows.

As a result, we have that for every $T$ positive

$$
\lim _{i \rightarrow \infty} \int_{\Sigma_{-1}^{i}} \Phi_{0, T}(\cdot,-1) d \mu=\lim _{i \rightarrow \infty} \int_{\Sigma_{0}^{i}} \Phi_{0, T}(\cdot, 0) d \mu,
$$

and so Huisken's monotonicity formula implies that

$$
\lim _{i \rightarrow \infty} \int_{-1}^{0} \int_{\Sigma_{i}^{s}}\left(|H|^{2}+\left|\mathbf{x}^{\perp}\right|^{2}\right) \exp \left(-|x|^{2}\right) d \mu d s=0
$$

because, due to (6), we can find a constant $C$ for which

$$
\begin{gathered}
\lim _{i \rightarrow \infty} \int_{-1}^{0} \int_{\Sigma_{i}^{s}}\left(|H|^{2}+\left|\mathbf{x}^{\perp}\right|^{2}\right) \exp \left(-|x|^{2}\right) d \mu d s=\lim _{i \rightarrow \infty} \int_{-1}^{0} \int_{\Sigma_{i}^{s}}|H|^{2} \exp \left(-|x|^{2}\right) d \mu d s \\
\leq \lim _{i \rightarrow \infty} C \int_{-1}^{0} \int_{\Sigma_{s}^{i}}\left|H+\frac{\mathbf{x}^{\perp}}{2(1-s)}\right|^{2} \Phi_{0,1} d \mu d s \\
=\lim _{i \rightarrow \infty} C\left(\int_{\Sigma_{-1}^{i}} \Phi_{0, T}(\cdot,-1) d \mu-\int_{\Sigma_{0}^{i}} \Phi_{0, T}(\cdot, 0) d \mu\right)=0 .
\end{gathered}
$$

We can then argue in the same way as was done at the end of page 471 and the beginning of page 472 in $[5$. (where $a=-1$ and $b=0$ ) to conclude that identity (31) holds for $s=0$.

Recall that $\Sigma_{0}^{i}$ converges as a Radon measure and as a current to a union of planes with possible multiplicities. Consider the complex-valued 2-form

$$
S=\lambda \wedge(\Omega\lrcorner J \mathbf{x}) .
$$

Because

$$
S_{\Sigma_{0}^{i}}=\left(\left|\mathbf{x}^{\perp}\right|^{2}+i\left\langle J \mathbf{x}, \mathbf{x}^{\top}\right\rangle\right) e^{i \theta} \operatorname{vol}_{\Sigma_{0}^{i}},
$$

we have

$$
\lim _{i \rightarrow \infty} \int_{\Sigma_{0}^{i} \cap B_{R}(0)}\left|\mathbf{x}^{\perp}\right|^{2} d \mu=0
$$

for every positive $R$. Moreover, the fact that $\Sigma_{0}$ is exact implies the existence of $\beta_{s}^{i}$ defined on $\Sigma_{s}^{i}$ for which

$$
d \beta_{s}^{i}=\lambda \quad \text { and } \quad\left|\nabla \beta_{s}^{i}\right|=\left|\mathbf{x}^{\perp}\right| .
$$


Hence, because $\Sigma_{0}^{i}$ is connected, we can apply Proposition B.1 and conclude the existence of $\bar{\beta}$ such that, after passing to a subsequence,

$$
\lim _{i \rightarrow \infty} \int_{\Sigma_{0}^{i} \cap B_{R}(0)}\left(\beta_{0}^{i}-\bar{\beta}\right)^{2} d \mu=0 .
$$

According to [5, Section 6], we have that

$$
\frac{d}{d s}\left(f_{s}^{i}\right)^{2}=\Delta\left(f_{s}^{i}\right)^{2}-2\left|\mathbf{x}^{\perp}-2 s H\right|^{2}, \quad \text { where } \quad f_{s}^{i}=\beta_{s}^{i}+2 s \theta_{s}^{i}-\bar{\beta},
$$

and so we can apply Huisken's monotonicity formula, Lemma 2.1] to the functions $f_{s}^{i}$ to conclude that, for every positive $T$,

$$
\lim _{i \rightarrow \infty} \int_{0}^{T} \int_{\Sigma_{s}^{i}} 2\left|\mathbf{x}^{\perp}-2 s H\right|^{2} \Phi_{0, T} d \mu d s \leq \lim _{i \rightarrow \infty} \int_{\Sigma_{0}^{i}}\left(\beta_{0}^{i}-\bar{\beta}\right)^{2} \Phi_{0, T} d \mu=0 .
$$

We want to show that, for every compactly supported function $\phi$ in $\mathbb{C}^{2}$,

$$
\frac{1}{s} \int_{\Sigma_{s}^{\infty}} \phi(x / \sqrt{s}) d \mu
$$

is constant as a function of $s$ for every $s>0$.

A standard computation shows that

$$
\begin{aligned}
\frac{d}{d s}\left(\frac{1}{s} \int_{\Sigma_{s}^{i}} \phi(x / \sqrt{s}) d \mu\right)=-\frac{1}{s^{2}} \int_{\Sigma_{s}^{i}} \phi & d \mu-\frac{1}{2 s^{5 / 2}} \int_{\Sigma_{s}^{i}}\langle D \phi, \mathbf{x}\rangle d \mu \\
+ & \frac{1}{s^{3 / 2}} \int_{\Sigma_{s}^{i}}\langle D \phi, H\rangle d \mu-\frac{1}{s} \int_{\Sigma_{s}^{i}} \phi|H|^{2} d \mu .
\end{aligned}
$$

Due to

$$
\Delta|x|^{2}=4+2\langle\mathbf{x}, H\rangle
$$

we obtain that

$$
2 \int_{\Sigma_{s}^{i}} \phi(x / \sqrt{s}) d \mu=-\int_{\Sigma_{s}^{i}}\langle\mathbf{x}, H\rangle \phi d \mu-\frac{1}{\sqrt{s}} \int_{\Sigma_{s}^{i}}\left\langle\mathbf{x}^{\top}, D \phi\right\rangle d \mu .
$$

Hence,

$$
\begin{aligned}
\frac{d}{d s}\left(\frac{1}{s} \int_{\Sigma_{s}^{i}} \phi(x / \sqrt{s}) d \mu\right)=\frac{1}{s^{3 / 2}} \int_{\Sigma_{s}^{i}}\left\langle D \phi, H-\frac{x^{\perp}}{2 s}\right\rangle d \mu & \\
& +\frac{1}{s} \int_{\Sigma_{s}^{i}} \phi\left\langle H, \frac{x^{\perp}}{2 s}-H\right\rangle d \mu .
\end{aligned}
$$

For every $0<a<b$ and $R>0$, we have that

$$
\int_{a}^{b} \int_{\Sigma_{s}^{i}}|D \phi|^{2} d \mu d s \text { and } \int_{a}^{b} \int_{\Sigma_{s}^{i} \cap B_{R}(0)}|H|^{2} d \mu d s
$$

are uniformly bounded. Therefore, (9) implies that

$$
\frac{1}{s} \int_{\Sigma_{s}^{\infty}} \phi(x / \sqrt{s}) d \mu
$$

is indeed independent of $s$ for all $s>0$, and this is equivalent to $\Sigma_{s}^{\infty}=\sqrt{s} \Sigma_{1}^{\infty}$ for all $s>0$. 
We now show the last property. Because $\Sigma_{1}^{\infty}$ has $\mathbf{x}^{\perp}=0$ and $\Sigma_{s}^{\infty}=\sqrt{s} \Sigma_{1}^{\infty}$ for all $s>0$, we obtain that $\mathbf{x}^{\perp}=0$ on $\Sigma_{s}^{\infty}$ for all $s$, and thus, from varifold convergence, we have for every $0<a<b$ and every $R>0$,

$$
\lim _{i \rightarrow \infty} \int_{a}^{b} \int_{\sum_{s}^{i} \cap B_{R}(0)}\left|\mathbf{x}^{\perp}\right|^{2} d \mu d s=0 .
$$

Hence, identity (9) combined with (10) implies that, for some universal constant $C$,

$$
\begin{gathered}
\lim _{i \rightarrow \infty} \int_{a}^{b} \int_{\Sigma_{s}^{i}}\left(|H|^{2}+\left|\mathbf{x}^{\perp}\right|^{2}\right) \exp \left(-|x|^{2}\right) d \mu d s \\
\quad=\lim _{i \rightarrow \infty} \int_{a}^{b} \int_{\Sigma_{s}^{i}}|H|^{2} \exp \left(-|x|^{2}\right) d \mu d s \\
\leq \lim _{i \rightarrow \infty} C \int_{a}^{b} \int_{\Sigma_{s}^{i}} 2\left|\mathbf{x}^{\perp}-2 s H\right|^{2} \exp \left(-|x|^{2}\right) d \mu d s \\
\quad \leq \lim _{i \rightarrow \infty} C \int_{a}^{b} \int_{\Sigma_{s}^{i}} 2\left|\mathbf{x}^{\perp}-2 s H\right|^{2} \Phi_{0,2 b} d \mu d s=0 .
\end{gathered}
$$

Thus, assuming without loss of generality that

$$
\int_{\Sigma_{1}^{i}}\left(|H|^{2}+\left|\mathbf{x}^{\perp}\right|^{2}\right) \exp \left(-|x|^{2}\right) d \mu d s=0
$$

we obtain from [5, Proposition 5.1] (which applies with no modifications) that $\Sigma_{1}^{\infty}$ is a union of Lagrangian planes with multiplicities. In order to prove the result, it suffices to show that $\Sigma_{1}^{\infty}$ equals $\Sigma_{0}^{\infty}$.

Huisken's monotonicity formula, Lemma 2.1. applied to (4) implies that for every $T>0$ and $0<s<T$,

$$
\int_{\Sigma_{0}^{\infty}} \Phi_{x_{0}, T} d \mu \leq \int_{\Sigma_{s}^{\infty}} \Phi_{x_{0}, T}(\cdot, s) d \mu=\int_{\Sigma_{1}^{\infty}} \Phi_{x_{0}, T}(\cdot, s) d \mu .
$$

Thus making $s$ converge to $T$ and then $T$ converge to zero, we obtain that

$$
\Theta_{0}\left(x_{0}\right) \geq \Theta_{1}\left(x_{0}\right),
$$

where $\Theta_{1}$ denotes the density function of $\Sigma_{1}^{\infty}$. Hence the support of $\Sigma_{1}^{\infty}$ is contained in the support of $\Sigma_{0}^{\infty}$.

Lemma 3.3. For every $x_{0}$ and $s<T$ we have

$$
\lim _{i \rightarrow \infty} \int_{\Sigma_{s}^{i}} \cos \left(\theta_{i, s}\right) \Phi_{x_{0}, T}(\cdot, s) d \mu=\lim _{i \rightarrow \infty} \int_{\Sigma_{s}^{i}} \cos \left(\theta_{i, s}\right) \Phi_{x_{0}, T}(\cdot, s) d \mu .
$$

Proof. The same reasoning that was used to show (5) shows the existence of $B$ so that

$$
\lim _{i \rightarrow \infty} \int_{0}^{s} \int_{\Sigma_{t}^{i}} 2|H|^{2} \Phi_{x_{0}, T} d \mu d t \leq B .
$$

Moreover, identity (10) readily implies the following analogue of (6):

$$
\lim _{i \rightarrow \infty} \int_{0}^{s} \int_{\Sigma_{t}^{i}}\left|\frac{\mathbf{x}^{\perp}}{2(T-t)}\right|^{2} \Phi_{x_{0}, T} d \mu d t=0 .
$$


With the help of (12) and (10), we can now apply the same reasoning used to show (17) in order to conclude that

$$
\lim _{i \rightarrow \infty}\left|\int_{0}^{s} \frac{d}{d t} \int_{\Sigma_{t}^{i}} \cos \theta_{i, t} \Phi_{x_{0}, T} d \mu d t\right| \leq \delta \frac{B}{2} .
$$

The result follows from making $\delta$ tend to zero.

Choosing $x_{0}$ to be in exactly one $P_{j_{0}}$ we use the above lemma to conclude that for any $0<s<T$,

$$
\begin{aligned}
& \int_{\Sigma_{1}^{\infty}} \cos \left(\bar{\theta}_{j}\right) \Phi_{x_{0}, T}(\cdot, s) d \mu=\lim _{i \rightarrow \infty} \int_{\Sigma_{s}^{i}} \cos \left(\theta_{i, s}\right) \Phi_{x_{0}, T}(\cdot, s) d \mu \\
&=\lim _{i \rightarrow \infty} \int_{\Sigma_{0}^{i}} \cos \left(\theta_{i, s}\right) \Phi_{x_{0}, T}(\cdot, 0) d \mu=\sum_{j=1}^{N} m_{j} \cos \left(\bar{\theta}_{j}\right) \int_{P_{j}} \Phi_{x_{0}, T}(\cdot, 0) d \mu \\
& \quad \geq \cos \left(\bar{\theta}_{j_{0}}\right) m_{j_{0}},
\end{aligned}
$$

where the last inequality follows from the almost-calibrated condition. Making $s$ converge to $T$, the almost-calibrated condition implies that

$$
\Theta_{0}\left(x_{0}\right) \leq \Theta_{1}\left(x_{0}\right) .
$$

\section{Graphical implies Flatness}

We start by defining what it means for a Lagrangian $L$ to have a graphical decomposition. Recall that $r_{0}$ was chosen (see Section 2) so that $\widehat{B}_{r_{0}}(x)$ is simply connected for all $x$ in $L$.

Definition 4.1. A Lagrangian $L$ is said to admit a graphical decomposition if, outside a compact set, $L$ can be decomposed into $N$ connected components $L_{j}$, $j=1, \ldots, N$, having the following property.

For each $j=1, \ldots, N$ there are constants $\bar{\theta}_{j}, R_{j}, S_{j}, n_{j}$ a positive integer, and a Lagrangian plane $P_{j}$,

$$
P_{j}=\left\{\left(u, 0, v \cos \bar{\theta}_{j}, v \sin \bar{\theta}_{j}\right) \mid(u, v) \in \mathbb{R}^{2}\right\},
$$

such that

(i) for every $x$ in $L_{j}, \widehat{B}_{r_{0}}(x)$ can be written as the graph of a function defined over $P_{j}$ with its derivatives bounded by $S_{j}$;

(ii) the projection of $L_{j}$ on $P_{j}$,

$$
\operatorname{Proj}_{P_{j}}: L_{j} \longrightarrow P_{j} \backslash B_{R_{j}}(0),
$$

is an $n_{j}$-fold covering map;

$$
\lim _{R \rightarrow \infty} \sup _{L_{j} \backslash B_{R}(0)}\left|\theta-\bar{\theta}_{j}\right|=0 .
$$

In particular, properties (i) and (iii) imply that

$$
\lim _{R \rightarrow \infty} \sup _{L \backslash B_{R}(0)}|H|=0 .
$$


Remark 4.2. In case $L$ is not embedded, property (ii) in Definition 4.1 should be interpreted as follows. If $F$ denotes the immersion of the surface $L$ in $\mathbb{C}^{2}$, then $\operatorname{Proj}_{P_{j}} \circ F$ is an $n_{j}$-fold covering map.

In this section we show that any translating solution $L$ to Lagrangian mean curvature flow which admits a graphical decomposition is a plane.

Theorem 4.3. If $L$ is a translating solution to Lagrangian mean curvature flow with uniformly bounded second fundamental form and admitting a graphical decomposition, then $L$ is a plane.

Proof. It suffices to show that the mean curvature of $L$ is zero because, in that case, we have from Proposition 2.2 that $L$ is a Special Lagrangian contained in some hyperplane and therefore a plane. The idea consists in finding a sequence of compact sets $K_{n}$ exhausting $L$ for which

$$
\lim _{n \rightarrow \infty} \oint_{\partial K_{n}}\left\langle\nu, e_{1}\right\rangle d \sigma=0,
$$

where $\nu$ denotes the exterior unit normal to $\partial K_{n}$ in $L$. The theorem follows because, due to Proposition 2.2 (ii),

$$
\oint_{\partial K_{n}}\left\langle\nu, e_{1}\right\rangle d \sigma=\int_{K_{n}}|H|^{2} d \mu .
$$

The following barrier will be needed to prove the main theorem.

Lemma 4.4. For every $\alpha<1 / 3$, there is a constant $R_{0}=R_{0}(\alpha)$ such that, for every constant $\delta$ and $B$, the function

$$
V_{\delta, B}=B|x|^{-\alpha} \exp (-|x| / 2)+\delta \exp \left(x_{1} / 2\right)
$$

satisfies

$$
\Delta V_{\delta, B} \leq V_{\delta, B} \frac{|H|^{2}+1}{4} \text { for all }|x| \geq R_{0} .
$$

Proof. Denote by $\partial_{r}, \partial_{r}^{\top}$, and $\partial_{r}^{\perp}$ the radial vector, its tangential projection on $L$, and its projection on the normal bundle of $L$, respectively. Set

$$
f(|x|)=|x|^{-\alpha} \exp (-|x| / 2)
$$

Away from the origin, we have

$$
\Delta|x|=\left\langle\partial_{r}, H\right\rangle+\frac{2}{|x|}-\frac{\left|\partial_{r}^{\top}\right|^{2}}{|x|},
$$

and so, because

$$
f^{\prime}=-\alpha \frac{f}{|x|}-\frac{f}{2} \quad \text { and } \quad f^{\prime \prime}=\alpha(\alpha+1) \frac{f}{|x|^{2}}+\alpha \frac{f}{|x|}+\frac{f}{4},
$$

we obtain that

$$
\begin{aligned}
\Delta f=f\left(\frac{\left|\partial_{r}^{\top}\right|^{2}}{4}-\frac{\left\langle\partial_{r}, H\right\rangle}{2}\right)+\frac{f}{|x|}\left(\alpha\left|\partial_{r}^{\top}\right|^{2}-1\right. & \left.-\alpha\left\langle\partial_{r}, H\right\rangle+\frac{\left|\partial_{r}^{\top}\right|^{2}}{2}\right) \\
& +\frac{f}{|x|^{2}}\left(\alpha(\alpha+2)\left|\partial_{r}^{\top}\right|^{2}-2 \alpha\right) .
\end{aligned}
$$


Hence

$$
\begin{aligned}
\Delta f & \leq f \frac{|H|^{2}+1}{4}+\frac{f}{|x|}\left(\frac{\alpha|H|^{2}}{2}+\alpha-\frac{1}{2}\right)+\frac{f}{|x|^{2}} \alpha^{2} \\
& \leq f \frac{|H|^{2}+1}{4}+\frac{f}{|x|} \frac{3 \alpha-1}{2}+\frac{f}{|x|^{2}} \alpha^{2} .
\end{aligned}
$$

Therefore, we can choose $R_{0}=R_{0}(\alpha)$ so that for all $|x| \geq R_{0}$ we have

$$
\Delta f \leq f \frac{|H|^{2}+1}{4}
$$

Proposition 2.2 (ii) implies that

$$
\Delta \exp \left(x_{1} / 2\right)=\exp \left(x_{1} / 2\right) \frac{2|H|^{2}+\left|e_{1}^{\top}\right|^{2}}{4}=\exp \left(x_{1} / 2\right) \frac{|H|^{2}+1}{4}
$$

and so the lemma follows.

This lemma implies the following decay for $\left|\theta-\bar{\theta}_{j}\right|$ on each component $L_{j}$, $j=1, \ldots, N$, given by the graphical decomposition.

Lemma 4.5. For each $\alpha<1 / 3$, there are constants $B$ and $R_{0}$ such that, on each component $L_{j}, j=1, \ldots, N$, we have

$$
\left|\theta(x)-\bar{\theta}_{j}\right|+|\nabla \theta|(x) \leq B|x|^{-\alpha} \exp \left(-|x| / 2-x_{1} / 2\right) \quad \text { for all } \quad|x| \geq R_{0} .
$$

Proof. For each $j=1, \ldots, N$, set

$$
u_{j}=\left(\theta-\bar{\theta}_{j}\right) \exp \left(x_{1} / 2\right)
$$

From Proposition 2.2 and (14) we know that

$$
\Delta u_{j}=u_{j} \frac{|H|^{2}+1}{4}+\exp \left(x_{1} / 2\right)\left(\Delta \theta+\left\langle e_{1}^{\top}, \nabla \theta\right\rangle\right)=u_{j} \frac{|H|^{2}+1}{4},
$$

and thus, we obtain from Lemma 4.4 that

$$
\Delta\left(V_{\delta, B}-u_{j}\right) \leq\left(V_{\delta, B}-u_{j}\right) \frac{|H|^{2}+1}{4} \text { for all }|x| \geq R_{0},
$$

where $R_{0}$ is chosen large enough so that $\partial L_{j} \subseteq B_{R_{0}}(0)$ and the constant $B$ is chosen so that, for every $j=1, \ldots, N$, we have

$$
x \in L_{j} \cap \partial B_{R_{0}}(0) \Longrightarrow B|x|^{-\alpha} \exp (-|x| / 2)>\left|u_{j}(x)\right| .
$$

Note that, for all $R$ sufficiently large, we have from Definition 4.1 (iii) that

$$
\sup _{L_{j} \cap \partial B_{R}(0)}\left|u_{j} \exp \left(-x_{1} / 2\right)\right| \leq \delta
$$

and thus

$$
\sup _{L_{j} \cap \partial B_{R}(0)}\left(V_{\delta, B}-u_{j}\right)>0 \quad \text { for every } j=1, \ldots, N .
$$

Applying the maximum principle to $L_{j} \cap\left(B_{R}(0) \backslash B_{R_{0}}(0)\right)$ for all $R$ sufficiently large, we have that

$$
u_{j}(x) \leq B|x|^{-\alpha} \exp (-|x| / 2)+\delta \exp \left(x_{1} / 2\right) \quad \text { for all } \quad|x| \geq R_{0} .
$$

As a result, after making $\delta$ tend to zero, we obtain

$$
\theta(x)-\bar{\theta}_{j} \leq B|x|^{-\alpha} \exp \left(-|x| / 2-x_{1} / 2\right) \quad \text { for all } \quad|x| \geq R_{0} .
$$


The correspondent estimate for $\left|\theta(x)-\bar{\theta}_{j}\right|$ follows in the same way by considering the function $V_{\delta, B}+u_{j}$. The estimate for $|\nabla \theta|$ is a consequence of Proposition 2.2 (i) and interior Schauder estimates.

The graphical decomposition implies the existence of $r_{1}$ so that, for every $p$ in $L_{j}$, $j=1, \ldots, N$, the projection of $\widehat{B}_{r_{0}}(p)$ on $P_{j}$ contains $B_{r_{1}}(\bar{p}) \cap P_{j}$, where $\bar{p}$ stands for the projection of $p$ on $P_{j}$. Thus, after an appropriate change of coordinates, a neighborhood of $p$ can be described as

$$
\left(u, v, \partial_{u} f, \partial_{v} f\right) \quad \text { with } \quad(u, v) \in B_{r_{1}}(\bar{p})
$$

for some function $f$ with $\mid$ Hess $f \mid$ uniformly bounded, where the coordinate $x_{1}$ equals $u$ and the coordinate $y_{1}$ equals $\partial_{u} f$. Furthermore, a direct computation shows that we can find a constant $D$ depending only on the constants $S_{j}$ (see Definition 4.1 (i)) for which one of the eigenvalues of Hess $f$ (called $\bar{\lambda}$ ) satisfies

$$
|\bar{\lambda}| \leq D\left|e_{1}^{\perp}\right|=D|\nabla \theta| \quad \text { on } \quad B_{r_{1}}(\bar{p}) .
$$

Thus, we obtain from Lemma 4.5 that, provided $|p|$ is large enough, one of the eigenvalues $\bar{\lambda}$ has the decay

$$
|\bar{\lambda}| \leq B|x|^{-\alpha} \exp (-|x| / 2-u / 2)
$$

for some constant $B$.

An explicit computation shows that the function $f$ satisfies the equation

$$
\arctan \lambda_{1}+\arctan \lambda_{2}=\theta(x)-\bar{\theta}_{j},
$$

where $\lambda_{1}$ and $\lambda_{2}$ are the eigenvalues of Hess $f$. As a result, Lemma 4.5 and estimate (15) imply that, provided $|p|$ is large enough,

$$
\mid \text { Hess }\left.f|\leq B| x\right|^{-\alpha} \exp (-|x| / 2-u / 2),
$$

where $\alpha<1 / 3$ and $B$ depends on $\alpha$.

On each of the connected components $L_{j}$, denote by $\gamma_{j, r}$ the lift to $L_{j}$ of the path on $P_{j}$ given by

$$
c_{j}(t)=(r \cos t, r \sin t) \quad 0 \leq t \leq 2 n_{j} \pi,
$$

where the variable $r$ will be made as large as we want.

There is $t_{0}=t_{0}\left(r, r_{1}\right)$ such that, for every $t_{1} \leq 2 n_{j} \pi$, we can find a function $f$ for which

$$
\gamma_{j, r}(t)=\left(r \cos t, r \sin t, \partial_{u} f, \partial_{v} f\right) \text { for all } t_{1}-t_{0} \leq t \leq t_{1}+t_{0},
$$

where, due to (16), the function $f$ restricted to this portion of $\gamma_{j, r}$ satisfies

$$
\mid \text { Hess } f \mid(t) \leq B r^{-\alpha} \exp (-r / 2-r \cos t / 2) \text {. }
$$

Using an obvious abuse of notation, the tangent vector $\gamma_{j, r}^{\prime}(t)$ is given by

$$
\gamma_{j, r}^{\prime}(t)=r\left(\partial_{t},(\operatorname{Hess} f)\left(\partial_{t}\right)\right) \text {, where } \partial_{t}=(-\sin t, \cos t),
$$

and, denoting by $\bar{\nu}$ the vector in $|x|^{2}$ for which

$$
\nu=(\bar{\nu},(\operatorname{Hess} f)(\bar{\nu})),
$$

then

$$
\left\langle\bar{\nu}, \partial_{t}+(\operatorname{Hess} f)^{*}(\operatorname{Hess} f)\left(\partial_{t}\right)\right\rangle=0 \quad \text { and } \quad 1=|\bar{\nu}|^{2}+|(\operatorname{Hess} f)(\bar{\nu})|^{2},
$$


where $(\operatorname{Hess} f)^{*}$ denotes the transpose of Hess $f$. Thus, provided we choose $r$ sufficiently large, we can find a constant $C$ such that

$$
\left|\bar{\nu}-\partial_{r}\right| \leq C r^{-2 \alpha} \exp (-r-r \cos t)
$$

and

$$
\left|\gamma_{j, r}^{\prime}(t)\right| \leq r\left(1+C r^{-2 \alpha} \exp (-r-r \cos t)\right)
$$

For this reason,

$$
\int_{t_{1}-t_{0}}^{t_{1}+t_{0}}\left\langle\nu, e_{1}\right\rangle\left|\gamma_{j, r}^{\prime}(t)\right| d t=\int_{t_{1}-t_{0}}^{t_{1}+t_{0}} r \cos t d t+Q
$$

where we can find a constant $C$ such that

$$
|Q| \leq \int_{t_{1}-t_{0}}^{t_{1}+t_{0}} C r^{1-2 \alpha} \exp (-r-r \cos t) d t .
$$

Therefore, we obtain from unique continuation that

$$
\begin{aligned}
\left|\oint_{\gamma_{j, r}}\left\langle\nu, e_{1}\right\rangle d \sigma\right| & \leq \int_{0}^{2 n_{j} \pi} C r^{1-2 \alpha} \exp (-r-r \cos t) d t \\
& =n_{j} C r^{1-2 \alpha} \int_{0}^{2 \pi} \exp (-r-r \cos t) d t
\end{aligned}
$$

Choose $\delta$ so that

$$
|\cos (y)+1| \leq(y-\pi)^{2} \quad \text { for all }|y-\pi| \leq \delta .
$$

Then, we can find a positive constant $D=D(\delta)$ so that, for all $r$ sufficiently large,

$$
\begin{aligned}
\int_{0}^{2 \pi} \exp (-r-r \cos t) d t & \leq \int_{\pi-\delta}^{\pi+\delta} \exp \left(-r(t-\pi)^{2}\right) d t+2 \pi \exp (-D r) \\
& \leq r^{-1 / 2} \int_{-\sqrt{r} \delta}^{\sqrt{r} \delta} \exp \left(-s^{2}\right) d s+2 \pi \exp (-D r) \\
& \leq D r^{-1 / 2}
\end{aligned}
$$

Hence, provided we choose $\alpha>1 / 4$ in Lemma 4.5, we have that

$$
\lim _{r \rightarrow \infty}\left|\oint_{\gamma_{j, r}}\left\langle\nu, e_{1}\right\rangle d \sigma\right|=\lim _{r \rightarrow \infty} r^{1 / 2-2 \alpha}=0 .
$$

Properties (i) and (ii) of Definition 4.1 imply that we can find a sequence of compact sets $K_{n}$ exhausting $L$ and such that

$$
\partial K_{n}=\gamma_{1, r_{n}} \cup \cdots \cup \gamma_{N, r_{n}},
$$

where $\left(r_{n}\right)_{n \in \mathbb{N}}$ is a sequence converging to infinity. This finishes the proof.

The next lemma gives conditions under which a translating solution $L$ satisfying $(H)$ admits a graphical decomposition.

Lemma 4.6. Assume that for almost all $-C_{1}<a<C_{1}$ we have

$$
\{\theta=a\} \subset B_{R(a)}
$$

for some positive $R(a)>0$. Then $L$ admits a graphical decomposition. 
Proof. For every $\alpha$, there is only one Lagrangian plane $P_{\alpha}$ with Lagrangian angle $\alpha$ and $\left|e_{1}^{\perp}\right|=0$ which is given by

$$
P_{\alpha}=\left\{\left.(u, 0, v \cos \alpha, v \sin \alpha)|(u, v) \in| x\right|^{2}\right\} .
$$

Set $\omega_{\alpha}$ to be the volume form of that plane extended by parallel translation to $\mathbb{C}^{2}$ and denote its Hodge-dual on $L$ by $* \omega_{\alpha}$.

We claim that for every $\varepsilon$, there is $\delta_{1}$ such that, for every $x$ in $L$ and every $\alpha$, we have

$$
\sup _{\widehat{B}_{r_{0}}(x)}|\theta-\alpha| \leq \delta_{1} \Longrightarrow \inf _{\widehat{B}_{r_{0}}(x)} * \omega_{\alpha} \geq \varepsilon
$$

A simple continuity argument shows the existence of $\delta_{2}$ such that if $Q$ is a Lagrangian plane with Lagrangian angle $\theta(Q)$, then for every $\alpha$

$$
\left|e_{1}^{\perp}\right| \leq \delta_{2} \quad \text { and }|\theta(Q)-\alpha| \leq \delta_{2} \quad \Longrightarrow \quad \omega_{\alpha}(Q) \geq \varepsilon,
$$

where $\omega_{\alpha}(Q)$ denotes the evaluation of $\omega_{\alpha}$ on $Q$. Moreover, as we argue next, we can find $\delta_{1} \leq \delta_{2}$ such that for all $x$ in $L$,

$$
\sup _{\widehat{B}_{r_{0}}(x)}|\theta-\theta(x)| \leq \delta_{1} / 2 \quad \Longrightarrow \quad \sup _{\widehat{B}_{r_{0}}(x)}|H| \leq \delta_{2} .
$$

If not, we could find a sequence of translating solutions $\left(T_{j}\right)_{j \in \mathbb{N}}$ converging smoothly on compact sets to another translating solution $T_{\infty}$ with Lagrangian angle constant on $\widehat{B}_{r_{0}}(0)$ and $|H|$ not identically zero on $\widehat{B}_{r_{0}}(0)$. This proves the desired claim.

Let $\left(x_{i}\right)_{i \in \mathbb{N}}$ be a sequence in $L$ with $\left|x_{i}\right|$ going to infinity and $\theta\left(x_{i}\right)$ converging to some $\alpha$. Set $\varepsilon=1 / 2$ in identity (18) and choose $\delta<\delta_{1} / 2$ so that

$$
\{\theta=\alpha \pm \delta\} \subset B_{R(\alpha \pm \delta)} .
$$

Set

$$
R=\max \{R(\alpha+\delta), R(\alpha-\delta)\}+2 \quad \text { and } \quad \Sigma=\{|\theta-\alpha| \leq \delta\} .
$$

We have

$$
\partial \Sigma \subseteq B_{R-2}(0)
$$

and if $x$ belongs to $\Sigma \backslash B_{R}(0)$, identity (18) implies that

$$
\inf _{\widehat{B}_{r_{0}}(x)} * \omega_{\alpha} \geq 1 / 2 \text {. }
$$

Moreover, from [5, page 476], there exists a constant $D$ such that

$$
\inf _{x \in L, r \leq r_{0}} r^{-2} \mathcal{H}^{2}\left(\widehat{B}_{r}(x)\right) \geq D^{-1}
$$

and so we can apply Lemma A.1 in order to conclude that, outside a compact set $K$,

$$
\Sigma \backslash K=L_{1} \cup \cdots \cup L_{N_{1}},
$$

where, for each $j=1, \ldots, N_{1}$,

$$
\operatorname{Proj}_{P_{\alpha}}: L_{j} \longrightarrow P_{\alpha} \backslash B_{R}(0)
$$

is an $n_{j}$-fold covering map and, for every $x$ in $\Sigma \backslash B_{R}(0), \widehat{B}_{r_{0}}(x)$ can be written as the graph of a function defined over $P_{\alpha}$ with its derivatives uniformly bounded.

Take an unbounded sequence $\left(y_{i}\right)$ in $L_{j}$ such that $\theta\left(y_{i}\right)$ converges to some $\bar{\theta}_{j}$ and suppose there is another unbounded sequence $p_{i}$ in $L_{j}$ such that $\theta\left(p_{i}\right)$ converges to some $\beta$ distinct from $\bar{\theta}_{j}$. Using the map $\operatorname{Proj}_{P_{\alpha}}$ and the local graphical property, we can find, for every $a$ between $\beta$ and $\bar{\theta}_{j}$, an unbounded sequence $w_{i}$ in $L_{j}$ such 
that $\theta\left(w_{i}\right)=a$. This contradicts our hypothesis and proves the third property of Definition 4.1. The first and second properties of Definition 4.1 follow because, by choosing $R_{j}>R$, we can replace $\alpha$ by $\bar{\theta}_{j}$ on each $L_{j}$.

We can repeat the whole process, but this time applied to $L \backslash \Sigma$. We only need to do this finitely many times because

$$
\lim _{R \rightarrow \infty} \mathcal{H}^{2}\left(L_{j} \cap B_{R}(0)\right) R^{-2} \geq C
$$

for some universal constant $C$.

\section{Proof of the MAin THEOREM}

We now prove Theorem A. We start by showing

Theorem 5.1. If $L$ is a translating solution that satisfies $(H)$, is almost-calibrated, and is static, then $L$ is a plane.

Proof. In view of Lemma 4.6 and Theorem 4.3 it suffices to show

Proposition 5.2. Let $L$ be a translating solution satisfying hypothesis $(H)$, almostcalibrated, and static. Then for almost all $-\pi / 2<a<\pi / 2$ we have

$$
\{\theta=a\} \subset B_{R(a)}
$$

for some positive $R(a)>0$.

Proof. The static condition implies the existence of a sequence of blow-downs

$$
L_{s}^{i}=\lambda_{i} L_{s / \lambda_{i}^{2}}, \quad \text { where } \quad \lim _{i \rightarrow \infty} \lambda_{i}=0,
$$

which converges weakly to

$$
L^{\infty}=m_{1} P_{1}+\cdots+m_{N} P_{N}
$$

for every $s \in \mathbb{R}$, where $P_{1}, \ldots, P_{N}$ are Lagrangian planes with multiplicities $m_{1}, \ldots, m_{N}$ and Lagrangian angles $\bar{\theta}_{1}, \ldots, \bar{\theta}_{N}$.

We claim that

$$
P_{j}=\left\{\left.\left(u, 0, v \cos \bar{\theta}_{j}, v \sin \bar{\theta}_{j}\right)|(u, v) \in| x\right|^{2}\right\} .
$$

The reason is that the coordinate $y_{1}$ equals, up to a constant, $-\theta$ (Proposition 2.2 (i)) and so is bounded for every $L_{t}$. Thus each plane $P_{j}$ must have $e_{1}^{\perp}=0$ because, for every $R>0$ and $s \leq 0$,

$$
\lim _{i \rightarrow \infty} \sup \left\{y_{1} \mid x \in L_{s}^{i} \cap B_{R}(0)\right\}=0 .
$$

Hence the almost-calibrated condition implies that $P_{j}$ is uniquely determined by its Lagrangian angle.

From Theorem 3.1 we know that the set of limiting Lagrangian angles does not depend on the sequence of rescalings chosen, and so any other convergent sequence of blow-downs $\left(\bar{L}_{s}^{i}\right)$ must converge to $L^{\infty}$ for every $s$. This observation will be used later.

Sard's Theorem implies that, for almost all $-\pi / 2<a<\pi / 2$, the set $\{\theta=a\}$ is a smooth submanifold of $L$ (possible empty). We argue that only finitely many curves contained in $\{\theta=a\}$ have finite length. Suppose that $\left(C_{j}\right)_{j \in \mathbb{N}}$ is a sequence of distinct curves contained in $\{\theta=a\}$ having finite length. Hypothesis $(H)$ implies that, for some $j_{0}$, we can find integers $b_{1}, \ldots, b_{j_{0}}$ such that

$$
b_{1}\left[C_{1}\right]+\cdots+b_{j_{0}}\left[C_{j_{0}}\right]=0,
$$


where $\left[C_{j}\right]$ denotes the homology class of $C_{j}$ in $H_{1}(L, \mathbb{Z})$. Thus, there is a compact set $K \subseteq L$ such that

$$
\partial K=b_{1} C_{1}+\cdots+b_{j_{0}} C_{j_{0}} .
$$

From Proposition 2.2 (i) we know that $\theta$ cannot have any interior maximum or minimum, and therefore, because $\theta$ equals $a$ on $\partial K, \theta$ must be constant on $K$. Analytic continuation implies that $\theta$ is constant on $L$, and this gives us a contradiction.

From (8) and (11) we can assume, without loss of generality, that after passing to a subsequence,

$$
\lim _{i \rightarrow \infty} \int_{L_{-1}^{i}}|H|^{2} \exp \left(-|x|^{2}\right) d \mu+\lim _{i \rightarrow \infty} \int_{L_{1}^{i}}|H|^{2} \exp \left(-|x|^{2}\right) d \mu=0 .
$$

Hence, from the coarea formula

$$
\begin{aligned}
& \int_{-\pi / 2}^{\pi / 2} \lambda_{i} \mathcal{H}^{1}\left(\{\theta=a\} \cap B_{\lambda_{i}^{-1}}\left(\lambda_{i}^{-2} e_{1}\right)\right) d a \\
&=\int_{-\pi / 2}^{\pi / 2} \mathcal{H}^{1}\left\{x \in L_{-1}^{i} \cap B_{1}(0) \mid \theta_{-1}^{i}=a\right\} d a=\int_{L_{-1}^{i} \cap B_{1}(0)}|H| d \mu \\
& \leq\left(\int_{L_{-1}^{i} \cap B_{1}(0)}|H|^{2} d \mu\right)^{1 / 2}\left(\mathcal{H}^{2}\left(L_{-1}^{i} \cap B_{1}(0)\right)\right)^{1 / 2},
\end{aligned}
$$

and this implies that, for almost all $-\pi / 2<a<\pi / 2$,

$$
\lim _{i \rightarrow \infty} \lambda_{i} \mathcal{H}^{1}\left(\{\theta=a\} \cap B_{\lambda_{i}^{-1}}\left(\lambda_{i}^{-2} e_{1}\right)\right)=0 .
$$

Likewise, we also obtain

$$
\lim _{i \rightarrow \infty} \lambda_{i} \mathcal{H}^{1}\left(\{\theta=a\} \cap B_{\lambda_{i}^{-1}}\left(-\lambda_{i}^{-2} e_{1}\right)\right)=0 .
$$

Choose $a$ distinct from $\bar{\theta}_{1}, \ldots, \bar{\theta}_{N}$ such that

$$
\begin{aligned}
\lim _{i \rightarrow \infty} \lambda_{i} \mathcal{H}^{1}\left(\{\theta=a\} \cap B_{\lambda_{i}^{-1}}\left(\lambda_{i}^{-2} e_{1}\right)\right) & \\
& \quad+\lim _{i \rightarrow \infty} \lambda_{i} \mathcal{H}^{1}\left(\{\theta=a\} \cap B_{\lambda_{i}^{-1}}\left(-\lambda_{i}^{-2} e_{1}\right)\right)=0
\end{aligned}
$$

and such that $\{\theta=a\}$ is a smooth submanifold of $L$.

It suffices to show that there is no connected curve $C$ with infinite length contained in $\{\theta=a\}$. If not, we could find an unbounded sequence $\left(x_{i}\right)$ in $C$ :

$$
x_{i}=t_{i} e_{1}+u_{i}, \quad \text { where } \quad\left|x_{i}\right|=\lambda_{i}^{-2} \quad \text { and } \quad\left\langle u_{i}, e_{1}\right\rangle=0 .
$$

\section{Lemma 5.3.}

$$
\liminf _{i \rightarrow \infty}\left|u_{i}\right|^{2}\left|t_{i}\right|^{-1}>0 .
$$

Proof. Suppose for some subsequence

$$
\lim _{i \rightarrow \infty}\left|u_{i}\right|^{2} t_{i}^{-1}=0 .
$$


Then

$$
\lim _{i \rightarrow \infty}\left|\lambda_{i}^{-2}-\right| t_{i}||=\lim _{i \rightarrow \infty}\left|t_{i}\right|\left|\sqrt{1+\left|u_{i}\right|^{2} t_{i}^{-2}}-1\right| \leq \lim _{i \rightarrow \infty}\left|t_{i}\right|^{-1}\left|u_{i}\right|^{2}=0
$$

and

$$
\lim _{i \rightarrow \infty} \lambda_{i}^{2}\left|x_{i}-t_{i} e_{1}\right|^{2}=\lim _{i \rightarrow \infty} \lambda_{i}^{2}\left|t_{i}\right|\left|u_{i}\right|^{2}\left|t_{i}\right|^{-1}=\lim _{i \rightarrow \infty} \frac{\left|t_{i}\right|}{\sqrt{t_{i}^{2}+\left|u_{i}\right|^{2}}}\left|u_{i}\right|^{2}\left|t_{i}\right|^{-1}=0 .
$$

Thus, for every $i$ sufficiently large, $x_{i}$ belongs to either

$$
B_{\lambda_{i}^{-1} / 2}\left(\lambda_{i}^{-2} e_{1}\right) \text { or } B_{\lambda_{i}^{-1} / 2}\left(-\lambda_{i}^{-2} e_{1}\right) \text {, }
$$

and so

$$
\mathcal{H}^{1}\left(C \cap B_{\lambda_{i}^{-1}}\left( \pm \lambda_{i}^{-2} e_{1}\right)\right) \geq \pi \lambda_{i}^{-1} .
$$

This contradicts identity (21).

After passing to a subsequence we can assume that

$$
\lim _{i \rightarrow \infty} t_{i}\left|u_{i}\right|^{-2}=s_{1} .
$$

Moreover, we also assume without loss of generality that

$$
\bar{L}_{s}^{i}=\left|u_{i}\right|^{-1} L_{s\left|u_{i}\right|^{2}}, \quad \text { where } \quad-\infty<s<\infty
$$

converges for all $s$, the sequence of manifolds $L_{i}=L-x_{i}$ converges to a smooth translating solution $L_{\infty}$ with $\theta_{\infty}(0)=a$, and $v_{i}=u_{i}\left|u_{i}\right|^{-1}$ converges to a vector $v$ perpendicular to $e_{1}$. The comments made at the beginning of this proof imply $\bar{L}_{s}^{i}$ converges to $L^{\infty}$ (given in (201) ) for every $s$.

For every $\beta, r>0$ and $s<0$,

$$
\begin{gathered}
\int_{L_{\infty}}\left(\theta_{\infty}-\beta\right)^{2} \Phi_{0, r}(\cdot, 0) d \mu=\lim _{i \rightarrow \infty} \int_{L}(\theta-\beta)^{2} \Phi_{x_{i}, r}(\cdot, 0) d \mu \\
=\lim _{i \rightarrow \infty} \int_{L_{-t_{i}}}(\theta-\beta)^{2} \Phi_{u_{i}, r-t_{i}}\left(\cdot,-t_{i}\right) \\
\leq \lim _{i \rightarrow \infty} \int_{L_{-t_{i}+s\left|u_{i}\right|^{2}}}(\theta-\beta)^{2} \Phi_{u_{i}, r-t_{i}}\left(\cdot,-t_{i}+s\left|u_{i}\right|^{2}\right) \\
=\lim _{i \rightarrow \infty} \int_{\bar{L}_{-t_{i}\left|u_{i}\right|^{-2}+s}(\theta-\beta)^{2}} \frac{\exp \left(-\frac{\left|x-v_{i}\right|^{2}}{4\left(r\left|u_{i}\right|^{-2}-s\right)}\right)}{4 \pi\left(r\left|u_{i}\right|^{-2}-s\right)} d \mu \\
=\int_{L_{s}^{\infty}}\left(\theta_{s-t_{0}}^{\infty}-\beta\right)^{2} \frac{\exp \left(\frac{|x-v|^{2}}{4 s}\right)}{-4 \pi s} d \mu \\
=\sum_{j=1}^{N} m_{j}\left(\bar{\theta}_{j}-\beta\right)^{2} \int_{P_{j}} \frac{\exp \left(\frac{|x-v|^{2}}{4 s}\right)}{-4 \pi s} d \mu .
\end{gathered}
$$

If $v$ did not belong to any $P_{j}$, we could make $s$ go to zero to conclude that the leftmost-hand side of the inequalities above is zero for every $\beta$, which is impossible. The fact that $v$ is perpendicular to $e_{1}$ implies that it can belong at most to one 
$P_{j_{0}}$, and thus, making $r$ go to zero and then $s$ go to zero, we see that

$$
(a-\beta)^{2} \leq m_{j_{0}}\left(\bar{\theta}_{j_{0}}-\beta\right)^{2}
$$

for every $\beta$. Therefore, $a=\bar{\theta}_{j_{0}}$, and this is a contradiction.

The proof of Theorem A will be completed after we show

Theorem 5.4. If $L$ is a translating solution that satisfies hypothesis $(H)$ and has

$$
\int_{L}|H|^{2} d \mu \leq C_{2}
$$

for some $C_{2}$, then $L$ is a plane.

Proof. In light of Lemma 4.6 and Theorem 4.3, it suffices to show that for almost all $-C_{1}<a<C_{1}$ we have

$$
\{\theta=a\} \subset B_{R(a)}
$$

for some positive $R(a)>0$. Moreover, we can argue as in Proposition 5.2 and see the fact that $L$ has a finite first Betti number implies that it is sufficient to show that for almost all $-C_{2} \leq s \leq C_{2}$ there is no curve contained in $\{\theta=s\}$ having infinite length.

Suppose that such a smooth curve exists and denote it by $C$. Then, for all $r$ sufficiently large, $C \cap\{|x|=r\}$ is not empty, and so

$$
\mathcal{H}^{1}(C \cap\{r \leq|x| \leq 2 r\}) \geq r .
$$

On the other hand, using the coarea formula, we have for all $r$,

$$
\begin{aligned}
\int_{-C_{1}}^{C_{1}} \mathcal{H}^{1}(\{\theta=s\} \cap\{r \leq|x| \leq 2 r\}) d s= & \int_{L \cap\{r \leq|x| \leq 2 r\}}|H| d \mu \\
\leq\left(\int_{L \cap\{r \leq|x| \leq 2 r\}}|H|^{2} d \mu\right)^{1 / 2} & \left(\mathcal{H}^{2}(L \cap\{r \leq|x| \leq 2 r\})\right)^{1 / 2} \\
& \leq \sqrt{C}_{1} r\left(\int_{L \cap\{r \leq|x| \leq 2 r\}}|H|^{2} d \mu\right)^{1 / 2} .
\end{aligned}
$$

Note that

$$
\lim _{r \rightarrow \infty} \int_{L \cap\{r \leq|x| \leq 2 r\}}|H|^{2} d \mu=0,
$$

and thus, for almost all $-C_{2} \leq s \leq C_{2}$, we can find a sequence $r_{i}$ going to infinity such that

$$
\lim _{i \rightarrow \infty} r_{i}^{-1} \mathcal{H}^{1}\left(\{\theta=s\} \cap\left\{r_{i} \leq|x| \leq 2 r_{i}\right\}\right)=0 .
$$

This contradicts (22). 


\section{Appendix A}

Let $\tau$ be the volume form of a plane $P$ extended by parallel translation to all of $\mathbb{C}^{2}$. We denote by $\operatorname{Proj}_{P}$ the projection onto the plane $P$ and assume that $P$ contains the line spanned by $e_{1}$. Given any surface $\Sigma$ in $\mathbb{C}^{2}$, we denote by $* \tau$ the Hodge-dual of $\tau$, and if $F$ denotes an immersion of $\Sigma$ on $\mathbb{C}^{2}$, we also use $\operatorname{Proj}_{P}$ to represent $\operatorname{Proj}_{P} \circ F$.

In what follows, $\Sigma$ will be a complete non-compact surface with smooth boundary such that $\widehat{B}_{r_{0}}(x)$ is simply connected for all $x$ in $L$,

$$
\sup _{R>0} R^{-2} \mathcal{H}^{2}\left(\Sigma \cap B_{R}(0)\right) \leq D \quad \text { and } \quad \inf _{x \in \Sigma, r \leq r_{0}} r^{-2} \mathcal{H}^{2}\left(\widehat{B}_{r}(x)\right) \geq D^{-1}
$$

for some constant $D$.

Lemma A.1. Assume that we can find $R>0$ and $\varepsilon>0$ such that

$$
\partial \Sigma \subset B_{R}(0)
$$

and

$$
\inf _{\widehat{B}_{r_{0}}(x)} * \tau \geq \varepsilon \text { for all } x \in \Sigma \backslash B_{R}(0) .
$$

Outside a compact set, $\Sigma$ can be decomposed into $N$ connected components $\Sigma_{j}$, $j=1, \ldots, N$, having the following property.

(i) For every $x$ in $\Sigma \backslash B_{R}(0), \widehat{B}_{r_{0}}(x)$ can be written as the graph of a function defined over $P$ with its derivatives bounded by a constant depending only on $\varepsilon$.

$$
\operatorname{Proj}_{P}: \Sigma_{j} \longrightarrow P \backslash B_{R}(0)
$$

is an $n_{j}$-fold covering map.

Proof. The first property is an immediate consequence of the fact that $\widehat{B}_{r_{0}}(x)$ is simply connected. Consider the map

$$
\operatorname{Proj}_{P}: \Sigma \backslash B_{R}(0) \longrightarrow P .
$$

The local graphical property combined with the uniform lower bounds on area densities implies that $\operatorname{Proj}_{P}^{-1}\left(B_{R}(0)\right)$ is a compact subset of $\Sigma \backslash B_{R}(0)$.

Decompose $\operatorname{Proj}_{P}^{-1}\left(P \backslash B_{R}(0)\right)$ into connected components $\left(\Sigma_{j}\right)_{j \in \mathbb{N}}$. The local graphical property implies the existence of an integer $n_{j}$ so that

$$
\operatorname{Proj}_{P}: \Sigma_{j} \longrightarrow P \backslash B_{R}(0)
$$

is an $n_{j}$-fold covering map with

$$
\operatorname{Proj}_{P}\left(\partial \Sigma_{j}\right) \subseteq \partial\left(P \backslash B_{R}(0)\right) .
$$

Moreover, there is a constant $C=C(\varepsilon)$ such that

$$
\lim _{R \rightarrow \infty} R^{-2} \mathcal{H}^{2}\left(\Sigma_{j} \cap B_{R}(0)\right) \geq C,
$$

and so there can only exist finitely many connected components $\Sigma_{j}$. 


\section{Appendix B}

The next proposition was proven in [5, Appendix A] with a slightly different hypothesis. For that reason we will only indicate the modifications in the proof.

Proposition B.1. Let $\left(N^{i}\right)$ and $\left(\alpha_{i}\right)$ be a sequence of smooth Lagrangian surfaces in $|x|^{4}$ and smooth functions on $N^{i}$, respectively, such that $\left(N^{i}\right)$ converges as Radon measure and as currents to a union of planes with positive integer multiplicities $N$. We assume that, for some $R>0$, the following properties hold:

a) There exists a constant $D_{0}$ such that

$$
\left.\mathcal{H}^{2}\left(N^{i} \cap B_{3 R}\right)\right) \leq D_{0} R^{2}
$$

and

$$
\cos \theta^{i} \geq D_{0}^{-1}
$$

for all $i \in \mathbb{N}$.

b)

$$
\lim _{i \rightarrow \infty} \int_{N^{i} \cap B_{3 R}(0)}\left|\nabla \alpha_{i}\right|^{2} d \mu=0 .
$$

c) There exists a constant $D_{1}$ for which

$$
\sup _{N^{i} \cap B_{3 R}(0)}\left|\nabla \alpha_{i}\right|+R^{-1} \sup _{N^{i} \cap B_{3 R}(0)}\left|\alpha_{i}\right| \leq D_{1}
$$

for all $i \in \mathbb{N}$.

d) For all $i \in \mathbb{N}$,

$$
N^{i} \cap B_{2 R}(0) \quad \text { is connected }
$$

and

$$
\partial\left(N^{i} \cap B_{3 R}(0)\right) \subset \partial B_{3 R}(0) .
$$

Then, there is a real number $\alpha$ such that, after passing to a subsequence, we have for all $\phi$ with compact support in $B_{R}(0)$ and all $f$ in $C(\mathbb{R})$,

$$
\lim _{i \rightarrow \infty} \int_{N^{i}} f\left(\alpha_{i}\right) \phi d \mu=f(\alpha) \mu_{N}(\phi)
$$

where $\mu_{N}$ denotes the Radon measure associated to $N$.

Proof. It suffices to find $\alpha \in \mathbb{R}$ and a sequence $\left(\varepsilon_{j}\right)$ converging to zero such that, for some appropriate subsequence, we have for all $j \in \mathbb{N}$

$$
\lim _{i \rightarrow \infty} \mathcal{H}^{2}\left(\left\{\left|\alpha_{i}-\alpha\right| \leq \varepsilon_{j}\right\} \cap B_{R}(0)\right)=\mathcal{H}^{2}\left(N \cap B_{R}(0)\right) .
$$

For the rest of this proof, $K=K\left(D_{0}, D_{1}, k\right)$ will denote a generic constant depending only on the mentioned quantities. Choose any sequence $\left(x_{i}\right)$ in $N^{i} \cap$ $B_{R}(0)$. After passing to a subsequence, we have that

$$
\lim _{i \rightarrow \infty} x_{i}=x_{0} \quad \text { and } \quad \lim _{i \rightarrow \infty} \alpha_{i}\left(x_{i}\right)=\alpha
$$

for some $x_{0} \in B_{R}(0)$ and $\alpha \in \mathbb{R}$. Furthermore, also consider a sequence $\left(\varepsilon_{j}\right)$ converging to zero such that, for all $j \in \mathbb{N}$,

$$
\lim _{i \rightarrow \infty} \mathcal{H}^{1}\left(\left\{\alpha_{i}=\alpha \pm \varepsilon_{j}\right\} \cap B_{3 R}\right)=0 .
$$


Such a subsequence exists because, by the coarea formula, we have

$$
\begin{aligned}
\lim _{i \rightarrow \infty} \int_{-\infty}^{\infty} \mathcal{H}^{1}\left(\left\{\alpha_{i}=s\right\} \cap B_{3 R}\right) d s= & \lim _{i \rightarrow \infty} \int_{N^{i} \cap B_{3 R}}\left|\nabla \alpha_{i}\right| d \mu \\
& \leq \lim _{i \rightarrow \infty} K R\left(\int_{N^{i} \cap B_{3 R}}\left|\nabla \alpha_{i}\right|^{2} d \mu\right)^{1 / 2}=0 .
\end{aligned}
$$

Define

$$
N^{i, \alpha, j}=\left\{\left|\alpha_{i}-\alpha\right| \leq \varepsilon_{j}\right\} \cap B_{3 R} .
$$

The Federer and Fleming Compactnes Theorem implies that, after passing to a subsequence, we have convergence to an integral current $\bar{N}^{\alpha, j}$ with no boundary on $B_{3 R}$. Its support is contained in a union of planes, and so the Constancy Theorem [7. Theorem 26.27] implies that $N^{\alpha, j}$ is a union of planes (with multiplicities) intersected with $B_{3 R}$.

The almost-calibrated condition implies that for every open set $B$,

$$
D_{0}^{-1} \mathcal{H}^{2}\left(N^{i, \alpha, j} \cap B\right) \leq \int_{N^{i, \alpha, j} \cap B} \operatorname{Re} \Omega \leq \mathcal{H}^{2}\left(N^{i, \alpha, j} \cap B\right) .
$$

Moreover, $N^{i}$ being almost-calibrated implies the existence (see [5, Lemma 7.1] of some constant $D$ such that

$$
\left(\mathcal{H}^{2}(A)\right)^{1 / 2} \leq D \mathcal{H}^{1}(\partial A)
$$

where $A$ is any open subset of $N$ with rectifiable boundary. Hence, [5, Lemma A.2] applies with no modification, and we conclude the existence of $K$ such that for every $j \in \mathbb{N}$,

$$
\mathcal{H}^{2}\left(N^{\alpha, j} \cap B_{R}\left(x_{0}\right)\right) \geq K R^{2} .
$$

Suppose that for some positive integer $j$ we have

$$
\mathcal{H}^{k}\left(N^{\alpha, j} \cap B_{R}(0)\right)<\mathcal{H}^{k}\left(N \cap B_{R}(0)\right) .
$$

Repeating the same type of argument, we can find $y_{0}$ in $B_{R}(0)$ and a closed interval $I$ disjoint from $\left[\alpha-\varepsilon_{j}, \alpha+\varepsilon_{j}\right]$ so that, after passing to a subsequence,

$$
\lim _{i \rightarrow \infty} \mathcal{H}^{2}\left(\alpha_{i}^{-1}(I) \cap B_{R}\left(y_{0}\right)\right) \geq K R^{2} .
$$

Given any positive integer $p$, pick disjoint closed intervals

$$
I_{1}, \ldots, I_{p}
$$

lying between $I$ and $\left[\alpha-\varepsilon_{j}, \alpha+\varepsilon_{j}\right]$. The connectedness of $N^{i} \cap B_{2 R}(0)$ implies that all $\alpha_{i}^{-1}\left(I_{l}\right) \cap B_{2 R}(0)$ are non-empty for $i$ sufficiently large. Hence, arguing as before, we find $y_{1}, \ldots, y_{p}$ in $B_{2 R}(0)$ such that, after passing to a subsequence,

$$
\lim _{i \rightarrow \infty} \mathcal{H}^{k}\left(\alpha_{i}^{-1}\left(I_{l}\right) \cap B_{R}\left(y_{l}\right)\right) \geq K R^{k}
$$

for all $l$ in $\{1, \ldots, p\}$. This implies that

$$
\begin{aligned}
\lim _{i \rightarrow \infty} \mathcal{H}^{2}\left(N^{i} \cap B_{2 R}(0)\right) & \geq \lim _{i \rightarrow \infty} \sum_{l=1}^{p} \mathcal{H}^{2}\left(\alpha_{i}^{-1}\left(I_{j}\right) \cap B_{R}\left(y_{l}\right)\right) \\
& \geq p K R^{2} .
\end{aligned}
$$

Choosing $p$ sufficiently large, we get a contradiction. 


\section{ACKNOWLEDGEMENTS}

The first author would like to express his gratitude to Yng-Ing Lee for some very stimulating discussions and to the referee for the extensive remarks which greatly improved the exposition.

\section{REFERENCES}

[1] G. Huisken, Asymptotic behavior for singularities of the mean curvature flow, J. Differential Geom. 31 (1990), 285-299. MR1030675 (90m:53016)

[2] T. Ilmanen, Elliptic Regularization and Partial Regularity for Motion by Mean Curvature. Mem. Amer. Math. Soc. 108 (1994), 1994. MR.1196160 (95d:49060)

[3] T. Ilmanen, Singularities of Mean Curvature Flow of Surfaces. Preprint.

[4] D. Joyce, Y.-I. Lee, M.-P. Tsui, Self-similar solutions and translating solitons for Lagrangian mean curvature flow, J. Differential Geom. 84 (2010), no. 1, 127-161. MR2629511 (2011f:53151)

[5] A. Neves, Singularities of Lagrangian Mean Curvature Flow: Zero-Maslov class case. Invent. Math. 168 (2007), 449-484. MR2299559 (2008d:53092)

[6] G. Perelman, The entropy formula for the Ricci flow and its geometric applications. preprint.

[7] L. Simon, Lectures on geometric measure theory, Proceedings of the Centre for Mathematical Analysis, Australian National University, 3. MR756417 (87a:49001)

[8] K. Smoczyk, Existence of solitons for the Lagrangian mean curvature flow. ETH Zürich; 1998; preprint.

[9] B. White, The size of the singular set in mean curvature flow of mean-convex sets. J. Amer. Math. Soc. 13 (2000), 665-695. MR1758759 (2001j:53098)

[10] B. White, The nature of singularities in mean curvature flow of mean-convex sets. J. Amer. Math. Soc. 16 (2003), 123-138 . MR1937202 (2003g:53121)

Department of Pure Mathematics, Imperial College London, South Kensington CamPus, London, SW7 2AZ, United Kingdom

E-mail address: aneves@imperial.ac.uk

Department of Mathematics, Fine Hall, Princeton University, Princeton, New JerSEY 08544

E-mail address: tian@math.princeton.edu 